\title{
Producción científica de la Revista de la Sociedad Española de Enfermería Nefrológica durante los años 2005-2008
}

\author{
Tomás Lozano-León ${ }^{1}$ - José Antonio Gómez-Fernández ${ }^{2}$ - Esther María Romero Indiano ${ }^{3}$ - Brígida Peral Belchior
}

${ }^{1}$ Unidad de Roting. ${ }^{2}$ Laboratorio de Análisis Clínicos.

${ }^{3}$ Distrito Sanitario Huelva-Costa, Dispositivo de Apoyo. Huelva. Servicio Andaluz de Salud (SAS), Huelva.

\section{Resumen}

Objetivo: Evaluar la actividad científica y la producción de la información de la Revista de la Sociedad Española de Enfermería Nefrológica (SEDEN), en el periodo 2005 a 2008, mediante el análisis bibliométrico.

Método: Estudio descriptivo transversal, de los resultados obtenidos del análisis de los artículos publicados en la Revista de la SEDEN. Los datos se obtuvieron mediante la consulta, a través de la Red, de la versión electrónica. Se tuvo en cuenta todas las tipologías documentales a excepción de las editoriales.

Resultados: Se publicaron un total de 131 artículos, siendo originales 75 (57,25\%). El área temática mayoritaria es la hemodiálisis. El número de autores es 4,44 por artículo. El perfil de autoría es una enfermera del ámbito asistencial de la Comunidad autónoma catalana 0 andaluza que utiliza 7,53 citas bibliográficas, con más de 7 años de antigüedad, por artículo original y utiliza metodología cuantitativa.

Conclusiones: Hay un incremento del $25 \%$ en el número de publicaciones con respecto al período 2001-2004.

\section{Correspondencia: \\ Tomás Lozano-León \\ C/ Maestro Salvador López, 38, 7 B \\ 21003 Huelva. \\ lozano.tomas@gmail.com}

PALABRAS CLAVE:

- BIBLIOMETRÍA

- ENFERMERÍA

- PUBLICACIONES CIENTÍFICAS

- INDICADORES BIBLIOMÉTRICOS

- INDICADORES DE PRODUCCIÓN CIENTÍFICA

\section{Scientific production of the Journal of the Spanish Association of Renal Nursing between the years 2005-2008}

\section{Abstract}

Objective: To assess the scientific activity and information production of the journal of the Spanish Association of Renal Nursing (SEDEN) for the period 2005-2008, by means of the bibliometric study.

Methods: Cross-seccional descriptive study of the results obtained from the analysis of the articles published in the journal of SEDEN. The data were obtained by consulting the electronic version through the web. All the documental possibilities were taken into account with the exception of editorials.

Results: A total of 131 articles were published, 75 $(57,25 \%)$ being original articles. Most of the articles are from hemodialysis area. The number of authors is 4.44 per article. The profile of authorship is an asistencial female nurse in the Catalan or Andalusian Comunity using 7,53 citations, with over 7 years old from the year of publication, for original article and quantitative methodology used.

Conclusions: There is a $25 \%$ increase in the number of publications for the period 2001-2004. 


\section{KEY WORDS:}

- BIBLIOMETRICS

- NURSING

- SCIENTIFIC PUBLICATIONS

- BIBLIOMETRIC INDICATORS

- SCIENTIFIC PUBLICATIONS INDICATORS

\section{Introducción}

La construcción de la Ciencia es un proceso complejo en el que la publicación del producto de su actividad es un hecho culminante, el resultado final de la actividad científica ${ }^{1,2}$. Sin embargo este resultado es parte de un fenómeno más amplio denominado ciclo de la producción científica, según el cual, la publicación permite a la comunidad científica de referencia conocer y contrastar los hallazgos ${ }^{1}$, incorporarla al acervo disciplinar $y_{\text {, }}$ en último lugar, utilizarla en la construcción teórica 0 práctica de la Ciencia mediante la cita y uso del documento en futuros productos ${ }^{3}$. La cita del documento por otros autores es el punto final del ciclo de la producción científica, y da lugar al inicio de un nuevo ciclo para el documento que se cita4.

El análisis de la publicación de artículos científicos es un eslabón fundamental dentro del proceso de investigación, se ha convertido en la unidad estándar que permite calificar la calidad del proceso generador de conocimiento y su impacto en el entorno científico ${ }^{5}$.

De la utilización cada vez más frecuente de métodos matemáticos y estadísticos para el análisis de la producción científica y de su calidad, nace la bibliometría definida por Garfield en 1978 como "la cuantificación de la información bibliográfica susceptible de ser analizada" 6,7. El uso de indicadores bibliométricos para analizar la actividad investigadora de un equipo científico, un área o un país, se basa en que las publicaciones científicas son un resultado esencial de dicha actividad. Un nuevo conocimiento adquiere valor cuando se da a conocer y se difunde, ya que es así como progresa la cienciå. La bibliometría es un instrumento esencial para evaluar el producto de la ciencia y en el caso de disciplinas incipientes en materia de investigación, como es la enfermería, es una herramienta esencial para determinar su avance y estado de consolidación ${ }^{9}$. Cuando una disciplina utiliza su conocimiento no hace sino identificarse con él, con sí misma, reconocerse en la herencia de su pasado y presente, configurarse en el presente y en el futuro ${ }^{10}$.
La Revista de la SEDEN inicia su andadura en 1998, como continuación de BISEAN (Boletín Informativo de la Sociedad Española de A.T.S. de Nefrología) durante los años 1978-1984 y posteriormente de BISEDEN (Boletín Informativo de la SEDEN) durante los años 1983-1997. Editada por la SEDEN tiene como objetivo servir de medio de consulta para los profesionales de enfermería que trabajan en el área renal, así como pacientes renales, familias y aquellos otros profesionales que muestran interés en la misma. Tiene una periodicidad trimestral y consta de un apartado científico y otro relacionado con las actividades de la Sociedad. Está disponible en formato papel y formato electrónico a través de su web (www.revistaseden. org). Se distribuye entre sus socios, colaboradores y bibliotecas. Está indizada en las bases de datos Cuiden, Cuidatge, BDIE, Latindex, IBECS, SIIC, Enfispo, CINAHL y Scielo.

Desde los inicios de esta revista se ha llevado a cabo un análisis bibliométrico de la misma en el período 2001$2004{ }^{9}$. Asimismo también se ha realizado un análisis de la producción científica de la Enfermería Nefrológica publicada a través de los diferentes Libros de Comunicaciones de los Congresos Nacionales de la SEDEN desde el año 1985 al 1993 11, en la que se analizan todos los artículos originales aceptados a comunicación oral en los citados congresos. Estos congresos suponen uno de los eventos principales de la SEDEN del cual nutre científicamente en gran medida las publicaciones posteriores de la Revista de la SEDEN.

El presente trabajo analiza las características de los artículos publicados en la Revista de la SEDEN entre los años 2005 y 2008.

\section{Material y métodos}

Diseño: Estudio bibliométrico, descriptivo transversal en el período de cuatro años (2005-2008).

Objetivo: Evaluar la actividad científica y la producción de la información de la Revista de la SEDEN mediante el análisis bibliométrico.

Población de estudio: Todos los artículos publicados en la Revista de la SEDEN en el período antes citado, excluidos las editoriales.

Recogida de datos: Los artículos se obtuvieron de la página web de la revista (www.revistaseden.org) y de cada artículo se extrajo, mediante una revisión manual, toda la información pertinente. 


\section{Indicadores:}

\section{Referente al artículo:}

- Identificación del artículo: año, volumen y número de la publicación.

- Tipo de artículo: original, carta al director, caso clínico, bibliografía comentada y artículo de revisión.

- Número de páginas.

- Área temática: hemodiálisis, diálisis peritoneal, trasplante, accesos vasculares y otros.

- En los artículos originales, tipo de metodología utilizada: cuantitativa, cualitativa y ambas.

\section{Referente a la autoría:}

- Número de autores por artículo.

- Sexo.

- Ámbito de trabajo: Asistencial, docente, gestión, investigador, mixto.

- Institución. Se tiene en cuenta la referida al primer firmante.

- Comunidad Autónoma de residencia del primer firmante.

- Autoría enfermera / Pluridisciplinar.

\section{Referente a las referencias bibliográficas:}

- Número de referencias.

- Antigüedad: media de las diferencias del año de publicación del artículo con cada una de las citas del mismo

Análisis de los datos: Se realiza un análisis descriptivo de todas las variables. Para la introducción y el análisis de los datos se utilizó el programa estadístico SPSS.

\section{Resultados}

\section{$\underline{\text { Referente al artículo }}$}

La Revista de la SEDEN, en los años analizados, está estructurada en volúmenes anuales de cuatro números cada uno con una periodicidad trimestral. Durante el período de estudio se publicaron un total de 131 artículos. Hay una gran homogeneidad productiva en cuanto al número de artículos por año. Se han contabilizado 33 artículos cada año a excepción de año 2006 que se redactaron 32. En cuanto a la tipología documental resaltar como tipo de artículo más frecuente el artículo original $(57,3 \%$ ) (figura 1 ). Se observa una similar distribución del tipo de artículos durante los cuatro años estudiados (figura 2). Se publica un artículo de revisión en los cuatro volúmenes analizados.

Más de la mitad de los artículos (54,20\%), eligen como tema principal de estudio la hemodiálisis o la diálisis peritoneal (figura 3). La extensión de los artículos oscila entre 1 y 11 páginas, con una media de 4,72 22,07. Casi la totalidad de los artículos originales utilizan metodología cuantitativa (93,33\%); un artículo utiliza conjuntamente análisis cuantitativo y cualitativo.

\section{Referente a la autoría}

Se han contabilizado un total de 539 autores para los 131 artículos analizados, de los cuales 389 (72\%) son mujeres. Lo que supone una media de autores que se sitúa en 4,44 2,58 por artículo, siendo 3 el número de autores que más se repite. Los autores son enfermeras del ámbito asistencial $113(86,26 \%)$ y en tan sólo 34 $(25,95 \%)$ de los artículos se realiza una colaboración pluridisciplinar.

\begin{tabular}{|c|c|c|c|c|c|c|c|}
\hline \multirow{2}{*}{\multicolumn{2}{|c|}{ Tipo Artículo }} & \multicolumn{6}{|c|}{$\mathrm{n}^{0}$ de referencias (agrupada) } \\
\hline & & $\leq 5$ & $6-10$ & $11-15$ & $16-20$ & $21+$ & Total \\
\hline \multirow{2}{*}{ Original } & Recuento & 14 & 36 & 12 & 9 & 4 & 75 \\
\hline & $\%$ & $18,70 \%$ & $48,00 \%$ & $16,00 \%$ & $12,00 \%$ & $5,30 \%$ & $100,00 \%$ \\
\hline \multirow{2}{*}{$\begin{array}{l}\text { Bibliografía } \\
\text { comentada }\end{array}$} & Recuento & 14 & 0 & 0 & 0 & 0 & 14 \\
\hline & $\%$ & $100,00 \%$ & $0,00 \%$ & $0,00 \%$ & $0,00 \%$ & $0,00 \%$ & $100,00 \%$ \\
\hline \multirow{2}{*}{$\begin{array}{l}\text { Carta al } \\
\text { Director }\end{array}$} & Recuento & 18 & 4 & 1 & 0 & 0 & 23 \\
\hline & $\%$ & $78,60 \%$ & $17,40 \%$ & $4,30 \%$ & $0,00 \%$ & $0,00 \%$ & $100,00 \%$ \\
\hline \multirow{2}{*}{ Caso clínico } & Recuento & 10 & 7 & 1 & 0 & 0 & 18 \\
\hline & $\%$ & $55,60 \%$ & $38,90 \%$ & $5,60 \%$ & $0,00 \%$ & $0,00 \%$ & $100,00 \%$ \\
\hline \multirow{2}{*}{$\begin{array}{l}\text { Artículo de } \\
\text { revisión }\end{array}$} & Recuento & 0 & 0 & 0 & 0 & 1 & 1 \\
\hline & $\%$ & $0,00 \%$ & $0,00 \%$ & $0,00 \%$ & $0,00 \%$ & $0,00 \%$ & $100,00 \%$ \\
\hline \multirow{2}{*}{ Total } & Recuento & 56 & 47 & 14 & 9 & 5 & 131 \\
\hline & $\%$ & $42,70 \%$ & $35,90 \%$ & $10,70 \%$ & $6,90 \%$ & $3,80 \%$ & $100,00 \%$ \\
\hline
\end{tabular}

Tabla 1: Número de referencias según tipo de artículo 


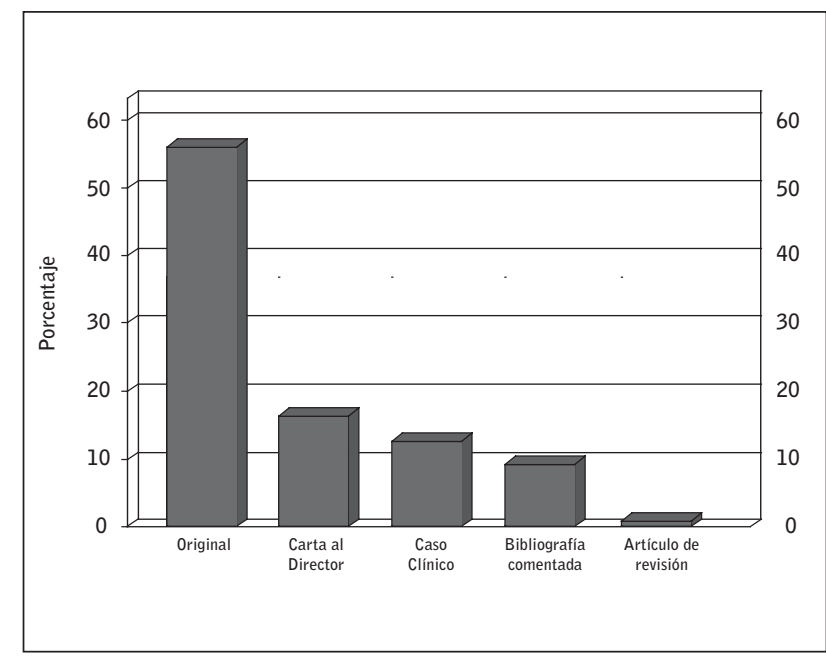

Figura 1: Distribición según tipología documental

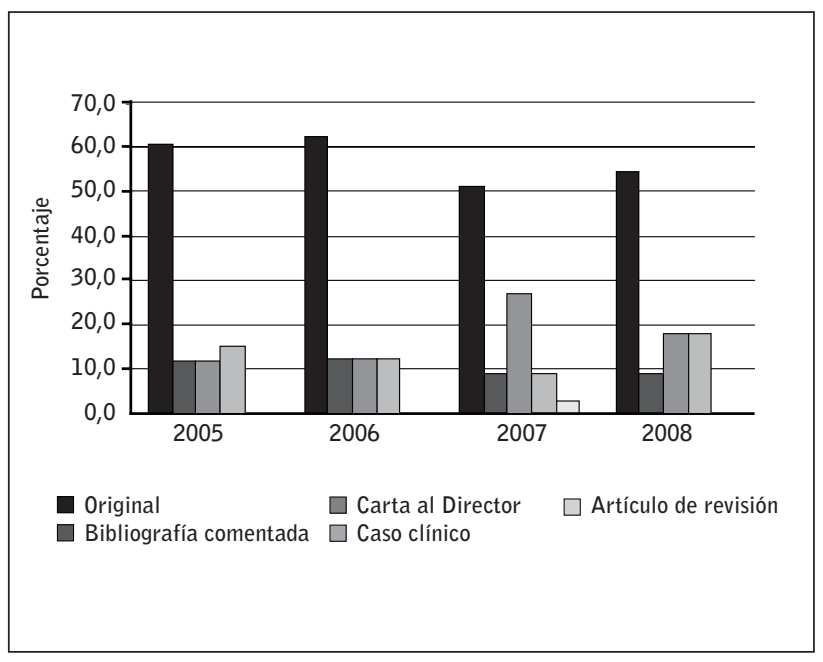

Figura 2: Evolución tipología documental por año

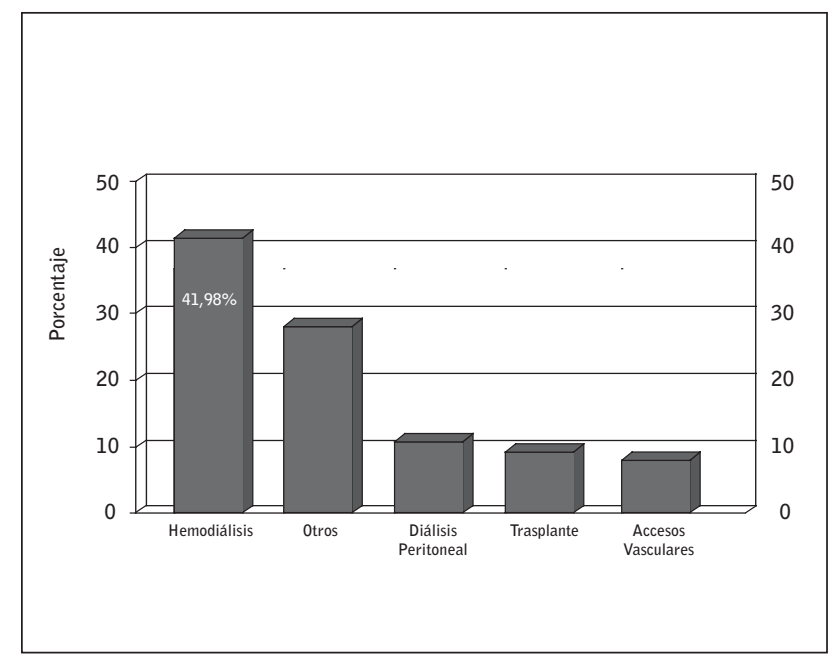

Figura 3: Distribución según área temática principal
Las comunidades autónomas que más han publicado en esta revista son las de Cataluña con $36(27,48 \%)$ publicaciones, seguidas por Andalucía con 20 (15,27\%) y Madrid con 19 (14,50\%), siendo las entidades más productivas la Fundació Pi i Sunyer de Bellvitge (Barcelona) con 17 artículos publicados (13,00\%) y el Hospital Universitario Virgen del Rocío (Sevilla) con 11 artículos publicado (8,40\%).

\section{Referente a las referencias bibliográficas}

La media de las referencias bibliográficas se sitúa en 7,53 referencias, siendo casi la mitad inferior 0 igual 5 referencias por artículo (tabla 1). La media de la distancia, en años (antigüedad) de la bibliografía referenciada en los artículos con respecto al año de publicación de los mismos se sitúa en más de 7 años (7,17 años) con un desviación típica de 3,5 años.

\section{Discusión}

Se observa un incremento de casi el $25 \%$ en el número de publicaciones con respecto al período 2001-2004\%, ya que se ha pasado de 108 a 131 artículos, lo cual nos indica que cada vez más, las enfermeras asumen la importancia que tiene la investigación como herramienta de trabajo para mejorar la práctica asistencial y calidad de vida de los pacientes.

Los temas tratados siguen siendo la Hemodiálisis y Diálisis peritoneal ${ }^{9}$ como principal problema de estudio, debido quizás a que estos estudios están realizados por enfermeras asistenciales que se enfrentan diariamente a estas técnicas y cuyas pequeñas modificaciones pueden suponer un gran beneficio para ellas y sobre todo para los pacientes.

Aunque la mayoría de los artículos son de metodología cuantitativa, se observa un ligero incremento de estudios cualitativos. Al ser enfermería una profesión con gran componente holístico, la investigación cualitativa se va introduciendo paulatinamente en nuestros estudios, siendo quizás la combinación de ambas metodologías lo más acertado.

Como ya se ha descrito en otros estudios bibliométri$\cos ^{12,13}$, las comunidades autónomas que más producción científica han aportado a ambas revistas son Cataluña, Andalucía y Madrid, por otra parte, son las que cuentan con mayor infraestructura hospitalaria absoluta y mayor número de centros de diálisis y, por tanto, es donde se atiende a un mayor número de enfermos. 


\section{Bibliografía}

1. Jiménez Contreras, E. Las revistas científicas: el centro y la periferia. Rev. Esp. Doc. Cient. 1992; 15(2):174-182.

2. López Piñero, JM; Terrada, ML. Los indicadores bibliométricos de la actividad médico-científica. (I) Usos y abusos de la bibliometría. Med. Clin. (Barc) 1992; 98:64-68.

3. Aleixandre, R; Giménez Sánchez, JV; Terrada, ML; López Piñero, JM. Análisis de consumo de información en la revista Medicina Clínica. Med Clin. (Barc) 1994;103:246-251.

4. Amezcua Martínez, M; Gálvez Toro, A; Cuesta de la Rosa, R; Heierle Valero, C; Poyatos Huertas, E. La Pequeña Ciencia. Producción, Repercusión y Transferencia del Conocimiento. La Enfermería del área Lingüística del Español y del Portugués. PI 03/0945. [citado el 20 de abril de 2009] Biblioteca Lascasas 2006; 2 (2). Disponible en: http://www.index-f.com/ lascasas/documentos/lc0139.php.

5. Rueda-Clausen Gómez CF, Villa-Roel Gutiérrez C, Rueda-Clausen Pinzón CE. Indicadores bibliométricos: origen, aplicación y nuevas propuestas. Med Unab. 2005; 8(1):29-36.

6. Camps D, Samar ME, Ávila RE, Recuero Y. Estudio bibliométrico de un volumen de la revista Archivos de Medicina. Arch Med [Revista en Internet] 2006. [citado el 20 de Abril de 2009]; 2(3). Disponible en: http: //archivosdemedicina.com.
7. Rousseau R. Indicadores bibliométricos y econométricos en la evaluación de instituciones científicas. CIMED. 2001; 9: 23-9.

8. Camps D. Estudio bibliométrico general de colaboración y consumo de la información en artículos originales de la revista Universitas Médica, período 2002 a 2006. Univ Med. 2007;48 (4):358-65.

9. Seguranyes G, Force E, Fuentelsaz C, Andreu L, Costa D. Características de los artículos publicados en las revistas "Matronas Profesión" y "Enfermería Nefrológica" durante los años 2001-2004. Rev Soc Esp Enferm Nefrol. 2006;9 (3):211-7.

10. Gálvez Toro, A. La legitimidad del valor de la producción científica enfermera. Index Enferm [revista en internet] 2007 [citado el 20 de abril de 2009]; 57. Disponible en: http://www.index-f.com/indexenfermeria/57/0708.php.

11. Crespo R, Rivero F. Perfil de la investigación original de enfermería nefrológica en los últimos nueve años. En: XVI Congreso nacional de la Sociedad Española de Enfermería Nefrológica, Madrid, 20-23 de Octubre de 1991. Barcelona: Hospal, S.A.; 1991. p.342-7.

12. Richart M. Estado de la producción científica de la enfermería española (y II). Index Enferm. 2000; 9(28-29): 15-8.

13. Cami J, Méndez-Vázquez R, Suñen Piñol E. Mapa bibliométrico de España 1994-2002: biomedicina y ciencias de la salud. Med Clin. Barcelona. 2005; 124(3):93-101. 